\title{
TRANSLATION AND CULTURAL ADAPTATION OF SHOULDER PAIN AND DISABILITY INDEX IN
} URDU (SPADI-U)

Karamat Ullah Keramat ${ }^{1}$, Muhammad Naveed Babur ${ }^{2}$, Patricia Mcloughlin ${ }^{3}$, Nimra Ilyas Bhutta ${ }^{1}$, Abdul Haseeb Bhutta $^{1}$, Pirzada Khattak ${ }^{1}$, Naila Kanwal ${ }^{1}$, Anam Habib ${ }^{1}$

\author{
Submitted: January 25, 2021 \\ Accepted: May 05, 2021 \\ Published: June 30, 2021
}

Authors' Affiliation
${ }^{1}$ Helping Hand Institute of
Rehabilitation
Mansehra
Isra Institute of Rehabilitation
Sciences, Isra University
Islamabad
${ }^{3}$ St. Patrick's Hospital Carrick-
on-Shannon, Ireland
Corresponding Author
Karamat Ullah Keramat
Helping Hand Institute of
Rehabilitation
Mansehra Sciences,
E-mail: karamatjee@yahoo.com

\begin{abstract}
Introduction: Shoulder joint is the third most-frequently involved structure in musculoskeletal conditions globally. More than 30 shoulder specific questionnaires have been generated in English in order to measure shoulder related quality of life and incidence of pain. Among all these questionnaires, Shoulder Pain and Disability Index (SPADI) is one of the most useful tools, therefore it has been translated, culturally adapted and validated to many languages. This study was carried out to translate and culturally adapt SPADI into Urdu language.
\end{abstract}

Material \& Methods: Study was conducted over a timeframe of three months, that's March 2019 to May 2019 in Helping Hand Institute of Rehabilitation Sciences, Mansehra. Translation and cultural adaptation of Shoulder Pain and Disability Index (SPADI) was carried out according to internationally published six-step guidelines. In accordance with the provided directions a number of changes were made to the six-step guidelines. Through consensus of the expert panel, its final version was field in and tested on forty individuals with and without shoulder pathologies in an equal proportion.

Results: Changes were required during each step of Beaton's guidelines for crosscultural adaptation. Translators during 'forward and backward translations' had faced a number of obstacles. Minor differences were found during reconciliation process and expert panel review. The pilot testing highlighted, misinterpretation among target population regarding item $3,5,7$ and 8 of disability scale due to cultural differences. These items were adjusted according to cultural relevance and conceptual equivalence to the original version. Final version was found relevant and easily understood by the patients having shoulder pathology.

Conclusion: SPADI-U has good face and content validity and it can be used to measure shoulder related quality of life in Urdu speaking population.

Key Words: Cross-Cultural Adaptation, Patient Reported Outcome measure, SPADI-U

\footnotetext{
The authors declared no conflict of interest and agreed to be accountable for all aspects of the work in ensuring that questions related to the accuracy or integrity of any part of the work are appropriately investigated and resolved. All authors contributed substantially to the planning of research, question designing, data collection, data analysis and writeup of the article.
}

This article may be cited as: Keramat KU, Babur MN, Mcloughlin P, Bhutta NI, Bhutta AH, Khattak P, Kanwal N, Habib A. Translation and cultural adaptation of shoulder pain and disability index in Urdu (SPADI-U). Rehman J Health Sci. 2021;3(1). 10-16

\section{INTRODUCTION}

Shoulder joint is the third most-frequently involved structure in musculoskeletal conditions globally and its frequency was found $11.4 \%$ at Helping Hand Institute of Rehabilitation Sciences, Pakistan. ${ }^{1}$ Prevalence of this condition is found very high in professions involving repeated use of the shoulder joint. For instance, nurses, dentists, teachers, house wives and others are at high risk of developing shoulder problems. ${ }^{2-4}$ Shoulder related pain affects quality of life and has a direct impact on the activities that constitute quality of life, such as housekeeping, ${ }^{5}$ overhead activities, ${ }^{6}$ recreational activities ${ }^{5}$ and sleep..$^{6,7}$

The impact of disease is assessed by various methods, ${ }^{8}$ including objective assessment ${ }^{6}$ which focuses on measurement of strength by manual muscle testing and range of motion by using goniometer. ${ }^{7-10}$ However, these measures are not related to outcome of the treatment ${ }^{6}$ and therefore, necessitate the use of Patient Reported Outcome (PRO) measures. ${ }^{8}$ Pain and pain related

disability is a subjective feeling and should be reported by the patients ${ }^{6,9}$ and therefore, structured subjective questionnaires are useful i.e PRO measures and this is the only way to collect specific information regarding their pain and disability. ${ }^{11}$ It is ,therefore, important for investigators and practitioners to use a valid and reproducible outcome measuring instrument to evaluate the functional status of patients. ${ }^{6,12}$

More than 30 shoulder specific questionnaires have been generated in English to measure shoulder related quality of life and pain. Among these, Disabilities of Arm Shoulder and Hand (DASH), Society Standardized Shoulder assessment form (SSS), the Western Ontario Shoulder Instability Index (WOSI), Shoulder Pain and Disability Index (SPADI), American Shoulder and Elbow Surgeon Standardized Assessment Form (ASES), Oxford Shoulder Test (OST), the Shoulder Disability Questionnaire (SDQ), Simple Shoulder Test (SST) and Constant (Murley) Score (CS) are commonly used. ${ }^{13}$ 
Among all these, SPADI is one of the most useful tool as it is a concise, valid and reliable subjective instrument which addresses most of the functional movements of the shoulder joint. ${ }^{11}$ It was developed by Roach et al. (1991) ${ }^{9}$ as a Visual Analogue Scale (VAS) and later on accepted as a Numeric scale for telephonic use and reliable results. ${ }^{14}$ Later on, it was modified and validated as a Numeric Rating Scale (NRS) by Williams et al. (1995). ${ }^{15}$ Acknowledging it's significance, SPADI has been translated, culturally adapted and validated to many languages, such as Chinese, ${ }^{6,16}$ German, ${ }^{17}$ Turkish, ${ }^{18}$ Spanish, ${ }^{8}$ Arabic ${ }^{19}$, Indian $\left(\right.$ Tamil $^{10}$, Hindi $^{20,21}$ and Telugu ${ }^{7}$ ), Greek, ${ }^{11,14}$ Dutch, ${ }^{12}$ Persian, ${ }^{22}$ Slovene, ${ }^{23}$ Brazilian, ${ }^{24}$ Danish, ${ }^{25}$ Thai, ${ }^{26}$ Korean, ${ }^{27}$ Norwegian, ${ }^{28}$ and Italian. ${ }^{29}$ However, until the time of current study SPADI was not translated or culturally adapted in Urdu language. Urdu is the national language of Pakistan and is spoken by $7 \%$ of the population as a first language; about $60 \%$ of the population use it as their second language ${ }^{30}$ in a total population of 224 Million people. ${ }^{31}$ Therefore, this study was aimed to translate and culturally adapt SPADI.

\section{MATERIAL AND METHODS}

Permission to translate SPADI to Urdu language was granted by the original author Kathryn E Roach on $7^{\text {th }}$ December 2017. The proposal for same was approved by the ethical approval committee of Helping Hand Institute of Rehabilitation Sciences. International guidelines proposed by Beaton el al. (2000) were followed in the translation and adaptation process (Figure 1). ${ }^{32}$ Two bilingual native speakers of Urdu and English language, one of whom (T1) a highly experienced physiotherapist, and the second (T2) an English lecturer at the National University of Modern Languages (NUML) Islamabad, took part in forward translation from the source language (English) to the target language (Urdu). The latter (T2) had no prior knowledge about the purpose of the translation. Both translators were required to translate conceptually/colloquially rather than literally, and to provide a written report of each item or sentence in relation to the difficulties in translation. A group of 8 professionals having the background of research and physical therapy then analysed the report provided by $\mathrm{T} 1$ and $\mathrm{T} 2$ interpreters. This team then provided a reconciled version of T12. Furthermore, later on T12 version was translated back to English language by an additional English language expert (T3) who knew both English and Urdu languages, however, had no prior knowledge of SPADI tool. The back translation provided by this expert was labelled as BT1 and BT2. An expert panel comprising of methodologists, physiotherapists and researchers reviewed the BT1, BT2 and T12 versions with the original version and a pre-final version of SPADI-U was agreed which had semantic, conceptual, experimental and idiomatic equivalence with the English version. Face validity of the pre-final version was determined by administering it to 40 individuals with an equal proportion of healthy subjects and those with shoulder pathology. Comment boxes were added at the end of the questionnaire on pre-final SPADI-U. Each subject was cognitively debriefed to probe what they thought about each questionnaire item and whether their chosen response was an accurate reflection of their individual pain/disability or not. A list of the questions being asked from the target group is presented in Table 1. Both the meaning of each item and the responses were explored. The adapted questions of the final version were then again field tested on 10 shoulder patients for face validity. This time no changes were required, therefore, the final SPADI-U was adopted.

\section{RESULTS}

In initial forward translation, the translators reported difficulty in translating and interpreting the words "Index", "Worst pain imaginable" and "at its worst". The word "Index" was translated as "Paimana (ييمان)" initially instead of "Isharia" (اشاري)) and the latter one was chosen as it was in common use and appropriate. For better understanding of pain and simplifying complex language terminologies, the interpreters agreed on a sit of words for a number of phrases, like for "worst pain imaginable" they will use "Shadeed Tareen Dard or Na Qabil-E- Bardasht Dard", which was translated in back translation as "excessive pain". Detailed comparison of each question in back translation has been explained in Table 2. Nevertheless, during stage 4 (harmonization), the expert panel stated that the word 'excessive' does not describe the pain severity, so the phrase "Na Qabil-EBardasht Dard" would create confusion among readers when compared to the original version. "Worst pain imaginable" created a challenge as "imaginable" may be translated as "Dard Ka Shadeed Tasawar" which again does not seem to be in semantic and colloquial equivalent of the questionnaire. Therefore, "Shadeed Tareen Dard" was acknowledged as the final phrase which shall be used. Item ' 1 ' in relation to severity "at its worst?" was discussed in detail. There was discrepancy in both forward translations. The phrase adopted through consensus was "Is Ki Shiddat Par" which later on was changed to "in its worst condition"/ "badtareen halat main". The colloquial equivalent of the "Worst" was "Bad Tareen" but 30 percent of the respondents during pre-testing were of the opinion that, "Bad Tareen" would already mean a score of 10 on NPR and recommended the phrase "Takleefdeh Halat Main". Thus, the recommendations on this question was adopted in prefinal version.

Words like "undershirt or jumper" in item three of disability scale and "pants" in item five in disability scale was replaced by Pakistani clothing/ dressing attire like "banyan ya banyan jaisa" and "shalwar ya pajama" in reconciliation. In item four of the disability scale, "shirt" was replaced by "Putting on something/shirts having buttons at the front" to make this item generalized for both male and female. Item eight of disability scale "removing something from your back pocket" was replaced and phrased as "reaching or removing something from the lower back or side pocket on the recommendation of $70 \%$ of the respondents in face validity. Similarly, "10 pounds" was replaced with "5 kg” or "ايك دهزَى". Back translation revealed similarity and equivalency between original and back translated version. Expert panel's review provided good evidence for content validity. These changes are summarized in Table 3 . The pretested version offered good face validity. All 40 respondents attempted every question by carefully reading and understanding. Question 1 in relation to pain severity was inappropriately understood by $30 \%$ of the participants. During debriefing of pretesting respondents, 
they confirmed that all questions were relevant to their condition, and that they performed the movement required by the item. About $37 \%$ of the patients were confused with the functional movement involved in item 3 of disability scale; as some of the females took this activity as hand behind back. Therefore, this item was revised to the required functional movement, as shown in table 3 . Almost $60 \%$ of the patients rated to item 5 of disability as 0 , with cognitive debriefing and pilot testing revealed that the patients with shoulder problem had no difficulty while putting on pants/ shalwar (loose pajamas). Instead, they had difficulty while removing pajamas/ shalwar. This aspect in Asian cultures is significantly different from the western culture. Therefore, this item was then culturally adapted as conceptual equivalent alternate, as shown in table 3 .

The question in relation to weightlifting was not clear to all respondents and was replaced with the phrase "weight carrying", as the Urdu word for lifting means both lifting and carrying. "Carrying" on the other hand involves swinging as well, and with consensus was adapted as "uthana".

The pre-final version was modified in order to generate the final version, which was then tested on 10 respondents. These respondents were cognitively debriefed for the questions being modified and cross checked by their responses. The respondents than reported the questionnaire as simple and effective. That's how final version of SPADI-U was created. Therefore, these patients were then included for the validation of the study.

\section{DISCUSSION}

The SPADI was successfully translated and culturally adapted for Urdu speaking population of Pakistan. The process of translation and cultural adaptation was done in accordance with the guidelines devised. ${ }^{32}$ The Urdu version of the SPADI questionnaire is simple, concise and has minimal cultural differences. It addresses most of the quality aspects of the local culture. Patient reported clinical outcomes are essential to evaluate patient's condition, therefore it can be used in research to evaluate success or failure of interventions. It requires minimum time around 2-5 minutes to be filled and completed by the patients. ${ }^{17}$

The culture of Muslim populations in general and that of Pakistan, specifically has marked differences with the western culture. These differences are in relation to clothing style, activities of daily living and religious practices. In this connection, about $60 \%$ of the participants reported no difficulty in putting on shalwar. It is obvious that putting on shalwar is a lot easier than putting on pants, since 'salwars' are made loser than pants. Therefore, cultural adaptation of SPADI for the Pakistani population was essential and questions such as "Taking off shalwar", which would need someone to make a greater effort, were modified.

The authors encountered challenges in both the word to word translation and in cultural adaptation of SPADI. It is important to mention that originality of the questions was maintained and substituted with appropriate words that were reflecting the original words used in SPADI.
Raising the hands in prayer or putting on a "qameez" is not duplicable to putting on a button shirt. The former requires a person to essentially abduct arm/or arms.

However, attempts in translation and adaptation have been made to conceptually adapt without much changes to semantic and colloquial equivalence. Similar attempts have been practiced in Arabic and Turkish version of SPADI translation. ${ }^{18,19}$

Certain terms used in Urdu version of SPADI are slightly different from the original scale. The terms replaced were "high shelf" with "high place", "pushing with involved arm" with "Pushing something with affected arm" in relation to the pain severity questions. The word "pant" was replaced with "shalwar", "shirt" with "anything that has buttons in front" " 10 pounds" with " $5 \mathrm{~kg}$ " 29 and "back pocket" with "purse or side pocket" in the disability section of SPADI. Similar changes were made to the original questionnaire e in consultation with the original author of SPADI. ${ }^{18,19,33}$

"Removing something from your lower back or back (or side) pocket?" has been modified in the other versions too, for example it was modified as "washing your lower back" in Arabic version. ${ }^{18,19}$ The addition of "side pocket" to this item was according to cultural context as most men wear 'shalwar qameez' having side pockets.

The translation and adaptation resulted in a version with good face and content validity. Modifications were compared with the previous literature of other translated versions. Cognitive debriefing (stage 5) revealed that adjustment was required in item 3 of disability scale to get the conceptual equivalence. "Putting on an undershirt/pullover/sweater or anything which requires overhead movement of hand?" was modified as was done previously in Brazilian version of SPADI in order to make it similar for both the genders.

Moreover, the pilot testing highlighted some activities of cultural concerns that were not covered in the SPADI, which may truly reflect quality of life of the local population. The activities like elevating hand during prayer (takbeer), covering the head with a scarf, holding overhead handles in public transports, washing and hanging the clothes, making bread, putting on caps/turbans, carrying weights overhead and other activities which constitute essential components of local quality of life and must be addressed in future researches and development of new questionnaires.

SPADI-U has good face and content validity. The present study translated the SPADI-U in the NRS version which is strongly recommended. ${ }^{10}$ Work on its reliability is in progress which will be reported once the study is completed.

\section{CONCLUSION}

SPADI has been successfully translated and culturally adapted according to the context of Pakistan into Urdu language. It has been pilot tested in a target population with and without shoulder pathology and has demonstrated good face and content validity.

\section{Acknowledgement}

We acknowledge Dr. Nazim Farooq and all those students and faculty who took part in this research and assist us in completion of this project.

\section{REFERENCES}


1. Keramat U, Unaiza B, Nimra M, Harris H, Awais Z. Frequency of patients presented with musculoskeletal disorders in Helping Hand institute of rehabilitation sciences, Mansehra (september 2014 - september 2018). 2019.

2. Mumtaz A, Khalid F, Ahmed A, Zahid H, Javaid HB, Islam F. Prevalence of Shoulder Pain Among School Teachers of Lahore, Pakistan. International Annals of Medicine. March, 2018;2(3):420. doi:https://doi.org/10.24087/IAM.2018.2.3.42 $\underline{0}$

3. Khan R, Ahmad F, Merchant MS. Prevalence of work related musculoskeletal disorders (MSD) among dentists. International Journal of Contemporary Medical Research. 2017;4(5):1208-1211.

4. Rathore FA, Attique R, Asmaa Y. Prevalence and perceptions of musculoskeletal disorders among hospital nurses in Pakistan: a cross-sectional survey.

Cureus. 2017;9(1):e1001. doi:https://dx.doi.org/10.7759\%2Fcureus. 1001 5. Croft P, Pope D, Silman A. The clinical course of shoulder pain: prospective cohort study in primary care. Primary Care Rheumatology Society Shoulder Study Group. BMJ: British Medical Journal. 1996;313(7057):601.

6. Yao M, Yang L, Cao Z-y, et al. Translation and cross-cultural adaptation of the Shoulder Pain and Disability Index (SPADI) into Chinese. Clin Rheumatol. 2017;36(6):1419-1426.

7. Gadam YK, Subramanian S, Patchava A, Kumar SC, Neerukonda SJ, Kambarthi N. Reliability and Validity of the Indian (Telugu) Version of the Shoulder Pain and Disability Index. Journal of Clinical \& Diagnostic Research. 2018;12(3):4.

8. Luque-Suarez A, Rondon-Ramos A, Fernandez-Sanchez M, Roach KE, MoralesAsencio JM. Spanish version of SPADI (shoulder pain and disability index) in musculoskeletal shoulder pain: a new 10-items version after confirmatory factor analysis. Health and quality of life outcomes. 2016;14(1):32.

9. Roach KE, Budiman-Mak E, Songsiridej N, Lertratanakul Y. Development of a shoulder pain and disability index. Arthritis \& Rheumatism: Official Journal of the
American College of Rheumatology. 1991;4(4):143-149.

10. Jeldi AJ, Aseer AL, Dhandapani AG, Roach KE. Cross-cultural adaption, reliability and validity of an Indian (Tamil) version for the Shoulder Pain and Disability Index. Hong Kong Physiotherapy Journal. 2012;30(2):99-104.

11. Vrouva S, Batistaki C, Koutsioumpa E, Kostopoulos D, Stamoulis E, Kostopanagiotou G. The Greek version of Shoulder Pain and Disability Index (SPADI): translation, cultural adaptation, and validation in patients with rotator cuff tear. $J$ Orthop Traumatol. 2016;17(4):315-326.

12. Thoomes-de Graaf M, Scholten-Peeters GG, Duijn E, Karel Y, Koes BW, Verhagen AP. The Dutch Shoulder Pain and Disability Index (SPADI): a reliability and validation study. Qual Life Res. 2015;24(6):1515-1519.

13. Angst F, Schwyzer H-K, Aeschlimann A, Simmen BR, Goldhahn J. Measures of adult shoulder function: Disabilities of the arm, shoulder, and hand questionnaire (DASH) and its short version (QuickDASH), shoulder pain and disability index (SPADI), American shoulder and elbow surgeons (ASES) society standardized shoulder assessment form, constant (Murley) score (CS), simple shoulder test (SST), oxford shoulder score (OSS), shoulder disability questionnaire (SDQ), and Western Ontario shoulder instability index (WOSI). Arthritis Care Res. 2011;63:S174S188.

14. Spanou A, Mamais I, Lamnisos D, Stasinopoulos D. Reliability and validity and of the Greek shoulder pain and disability index in patients with shoulder pain. Disabil Rehabil. 2019:1-6.

doi:10.1080/09638288.2018.1519728

15. Williams Jr JW, Holleman Jr DR, Simel D. Measuring shoulder function with the Shoulder Pain and Disability Index. The Journal of rheumatology. 1995;22(4):727-732. 16. Wang W, Jia Z-y, Liu J, et al. Crosscultural adaptation and validation of the Chinese version of the shoulder pain and disability index in patients with symptomatic shoulder pain: A prospective case series. Medicine. 2018;97(26)

17. Angst F, Goldhahn J, Pap G, et al. Cross-cultural adaptation, reliability and validity of the German Shoulder Pain and 
Disability Index (SPADI). Rheumatology (Oxford). 2006;46(1):87-92.

18. Bumin G, Tüzün EH, Tonga E. The Shoulder Pain and Disability Index (SPADI): cross-cultural adaptation, reliability, and validity of the Turkish version. $J$ Back Musculoskelet Rehabil. 2008;21(1):57-62.

19. Alsanawi HA, Alghadir A, Anwer S, Roach KE, Alawaji A. Cross-cultural adaptation and psychometric properties of an Arabic version of the Shoulder Pain and Disability Index. Int $J$ Rehabil Res. 2015;38(3):270-275.

20. Sharma S, Hussain ME. Psychometric Properties of the Hindi Version of SPADI in Overhead Athletes With Shoulder Impingement Syndrome. International Journal of Athletic Therapy and Training. 2018;23(6):246-252.

21. Sharma N, Sharma S, Kataria C. Translation and Adaptation of Shoulder Pain and Disability Index (SPADI) into Hindi-Part 1. Ind J Physiother Occup Ther. 2011;5(4):142145.

22. Ebrahimzadeh MH, Birjandinejad A, Golhasani F, Moradi A, Vahedi E, Kachooei AR. Cross-cultural adaptation, validation, and reliability testing of the Shoulder Pain and Disability Index in the Persian population with shoulder problems. Int $J$ Rehabil Res. 2015;38(1):84-87.

23. Jamnik H, Spevak MK. Shoulder pain and disability index: validation of Slovene version. Int J Rehabil Res. 2008;31(4):337-341. 24. Martins J, Napoles BV, Hoffman CB, Oliveira AS. The Brazilian version of Shoulder Pain and Disability Index: translation, cultural adaptation and reliability. Brazilian Journal of Physical Therapy. 2010;14(6):527-536.

25. Christiansen DH, Andersen JH, Haahr JP. Cross-cultural adaption and measurement properties of the Danish version of the Shoulder Pain and Disability Index. Clin Rehabil. 2013;27(4):355-360.
26. Phongamwong C, Choosakde A. Reliability and validity of the Thai version of the Shoulder Pain and Disability Index (Thai SPADI). Health and quality of life outcomes. 2015;13(1):136.

27. Seo H, Lee K, Jung K, Chung Y. Reliability and validity of the Korean version of shoulder pain and disability index. J Spec Educ Rehabil Sci. 2012;51(2):319-36.

28. Tveitå EK, Ekeberg OM, Juel NG, Bautz-Holter E. Responsiveness of the shoulder pain and disability index in patients with adhesive capsulitis. BMC Musculoskelet Disord. 2008;9(1):1-8.

29. Marchese C, Cristalli G, Pichi B, et al. Italian cross-cultural adaptation and validation of three different scales for the evaluation of shoulder pain and dysfunction after neck dissection: University of California-Los Angeles (UCLA) Shoulder Scale, Shoulder Pain and Disability Index (SPADI) and Simple Shoulder Test (SST). Acta Otorhinolaryngol Ital. 2012;32(1):12.

30. Dar M, Anwaar H, Vihman M, KerenPortnoy T. Developing an Urdu CDI for early language acquisition. York Papers in Linguistics. 2015;14(2):1-14.

31. worldometer. Pakistan Population. Updated April 25, 2021,. Accessed April 24, 2021,

2021.

https://www.worldometers.info/world-

population/pakistan-population/

32. Beaton DE, Bombardier C, Guillemin F, Ferraz MB. Guidelines for the process of cross-cultural adaptation of self-report measures. Spine. 2000;25(24):3186-3191.

33. Torres-Lacomba M, Sánchez-Sánchez B, Prieto-Gómez V, et al. Spanish cultural adaptation and validation of the shoulder pain and disability index, and the oxford shoulder score after breast cancer surgery. Health and quality of life outcomes. 2015;13(1):63. 


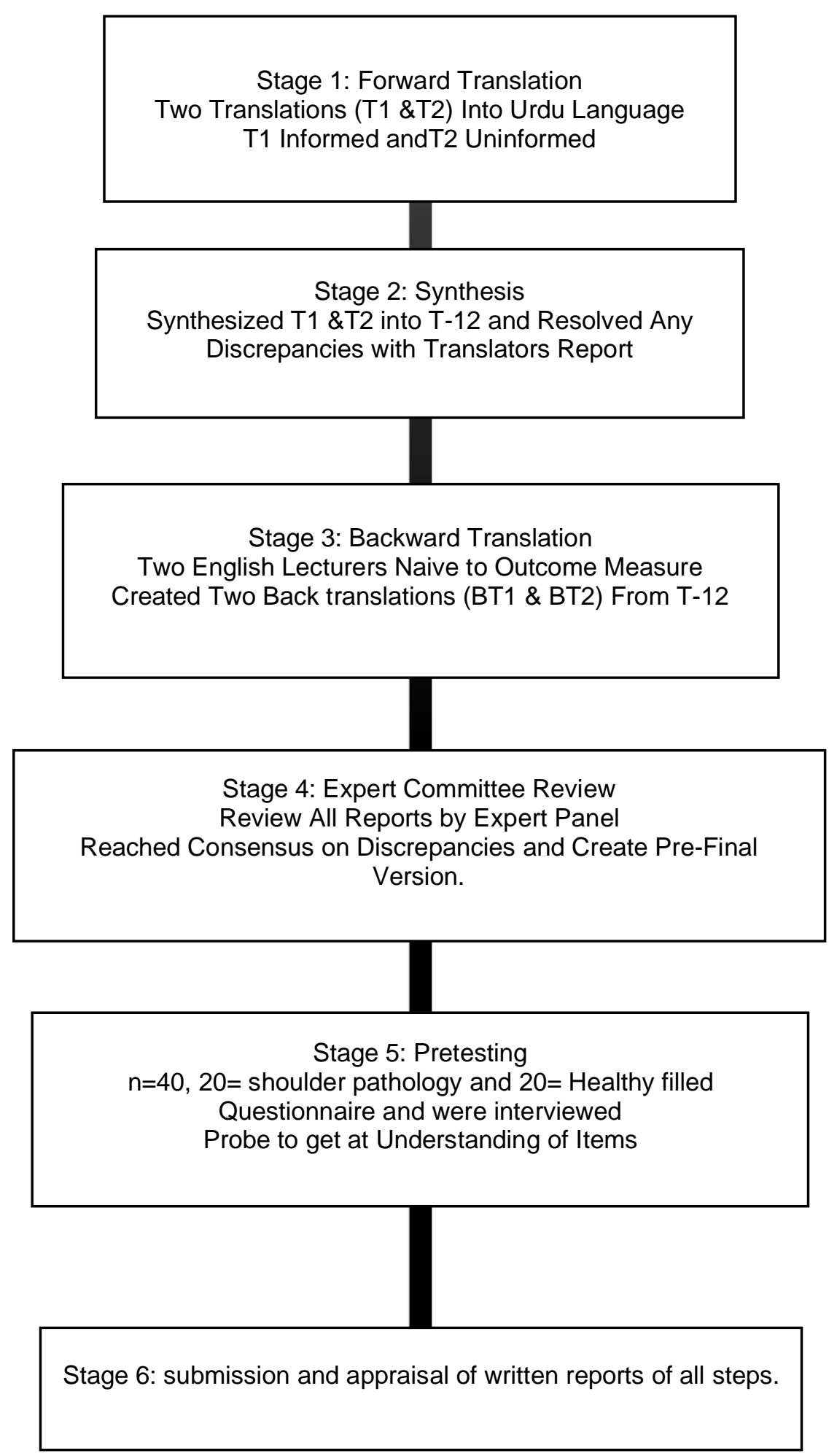

Figure 1: Steps of Translation and Cultural Adaptation of a PRO Measure 
Table 1: List of Questions asked during the interview of the Subjects (respondents)

\begin{tabular}{|ll|}
\hline $\mathbf{1}$ & Do you think SPADI-U is relevant to your shoulder condition? \\
$\mathbf{2}$ & Do you have any suggestions for words which create ambiguity for you? \\
$\mathbf{3}$ & Have you related the below questions with the above question asking about the severity \\
$\mathbf{4}$ & of pain and disability? \\
$\mathbf{5}$ & What did you think the question was asking about? \\
$\mathbf{6}$ & Could you repeat the question in your own words? \\
$\mathbf{7}$ & Could you perform the specific movement of the shoulder as required in the question? \\
\hline
\end{tabular}

Table 2: Original Version of SPADI and Back Translation of SPADI-U in English

\begin{tabular}{|ll|}
\hline Original SPADI & BT SPADI-U (T12) \\
\hline Pain & Pain \\
At its worst? & In worst condition? \\
When lying on involved side? & When lying on affected side? \\
Reaching for something on a higher shelf? & Reaching for something on a higher place? \\
Touching the back of your neck? & Touching the back of your neck? \\
Pushing with the involved arm? & Pushing with the affected arm? \\
\hline Disability & Disability \\
Washing your hair? & Washing your hair? \\
Washing your back? & Washing your back? \\
Putting an undershirt or jumper? & Putting on something/shirts having button at front? \\
Putting on your pants? & Putting on trousers and pants? \\
Placing an object on a high shelf? & Placing something at a higher place? \\
Carrying a heavy object of 10 pound (4.5kg?) & Lifting weight of 5 kg? \\
Removing something from your back pocket? & Getting something from your side pocket or shoulder \\
& bag? \\
\hline
\end{tabular}

Table 3: List of Items that were adjusted during Cross-Cultural Translation

\section{Items Requiring Adjustment}

D3: Putting on undershirt or jumper

D5: Putting on your pant?

D7: Carrying a heavy object of 10 pounds (4.5 kilograms))

D8: Removing something from your back pocket'

\section{Culturally Adapted}

Putting on an undershirt/pullover sweater or anything which requires overhead movement of hand?

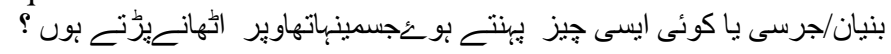
Putting on or removing your pajama/shalwar?

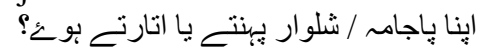

Carrying or lifting a weight of 5 kilogram?

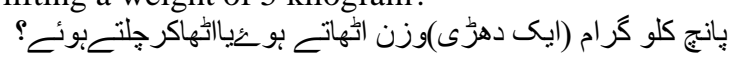

Removing something from your lower back or back (or side) pocket?'

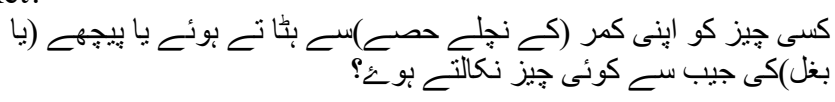

